\title{
TUNABILITY OF THE NLC FINAL FOCUS SYSTEM*
}

\author{
Pantaleo Raimondi, Andrei Seryi, Peter Tenenbaum \\ SLAC, Stanford, USA
}

\begin{abstract}
The performance of the new NLC Final Focus system has been investigated as a function of the incoming beam characteristics, such as energy, emittances, energy spread. A preliminary study of the tolerances and the tunability of the system is presented in this paper.
\end{abstract}

\section{FINAL FOCUS OPTICS}

The NLC Final Focus (FF) Design is based on the concept of local chromaticity correction [1]. After further optimization, the layout of the FF is shown in Fig.1, where for completeness, the collimation section is also included. The main differences with respect to the optics described in [1] are:

- A quadrupole triplet between the $\mathrm{X}$ and $\mathrm{Y}$ 'geometric sextupoles' provides the optimal phase relation between these sextupoles and the 'chromatic sextupoles' in the Final Doublet (FD).

- A bend between the $\mathrm{X}$ and $\mathrm{Y}$ geometric sextupoles provides a more local chromatic correction and a better cancellation of the second order dispersion.

- Two decapoles, separated by an 'Identity Transformation', in between the $\mathrm{X}$ and $\mathrm{Y}$ geometric sextupoles, cancels $4^{\text {th }}$ order chromo-geometric aberrations.

- The collimation section is modified to reduce its chromatic aberrations, which are now cancelled in the Final Focus itself. The two systems have now been designed and optimized simultaneously.

- Octupole doublets [2] provide non-linear 'halo folding'.

With these modifications, the bandwidth and the dynamic aperture of the Beam Delivery System (BDS) have been greatly improved with respect to [1]. The strength of all the quadrupoles (except the FD) has been decreased, relaxing the tolerances and improving the tunability of the system.

\section{FINAL FOCUS PERFORMANCE}

The FF performance have been studied as a function of the incoming beam characteristics. The bandwidth of the system, based on the NLC design parameters listed in Tab.1 is shown in Fig.2. Fig.3 shows the dynamic aperture for an incoming beam with emittances more than $10^{4}$ larger than the design values. Fig. 4 shows the luminosity as a function of energy, assuming constant normalized emittances for the NLC parameters, and also using the CLIC [3] parameters. In this BDS design, it is

*Work supported by the U.S. Department of Energy, Contract DE-AC03-76SF00515 also possible to rescale the bends so as to achieve the best luminosity performance at any given energy, while leaving the IP position unchanged.

Fig. 5 and 6 show the luminosity as a function of the IP $\beta$ functions and the incoming emittances. The system is capable of delivering a luminosity higher than required for the NLC design, with smaller IP $\beta$ 's or emittances. This leaves headroom for possible upgrades of the NLC performance once it is in operation.

Table 1: Beam parameters

\begin{tabular}{|c|c|c|c|}
\hline Beam energy & & $(\mathrm{GeV})$ & 500 \\
\hline Normalized emittances & $\gamma \varepsilon_{x} / \gamma \varepsilon$ & $(\mu \mathrm{m})$ & $3.6 / 0.035$ \\
\hline IP Beta functions & $\beta_{x} / \beta_{y}$ & $(\mathrm{~mm})$ & $10 / 0.12$ \\
\hline IP Beam sizes & $\sigma_{x} / \sigma_{y}$ & $(\mathrm{~nm})$ & $190.0 / 2.0$ \\
\hline IP Beam divergence & $\theta_{x} / \theta_{y}$ & $(\mu \mathrm{rad})$ & $19.0 / 17.5$ \\
\hline Energy spread & & $\left(10^{-3}\right)$ & 3.0 \\
\hline
\end{tabular}

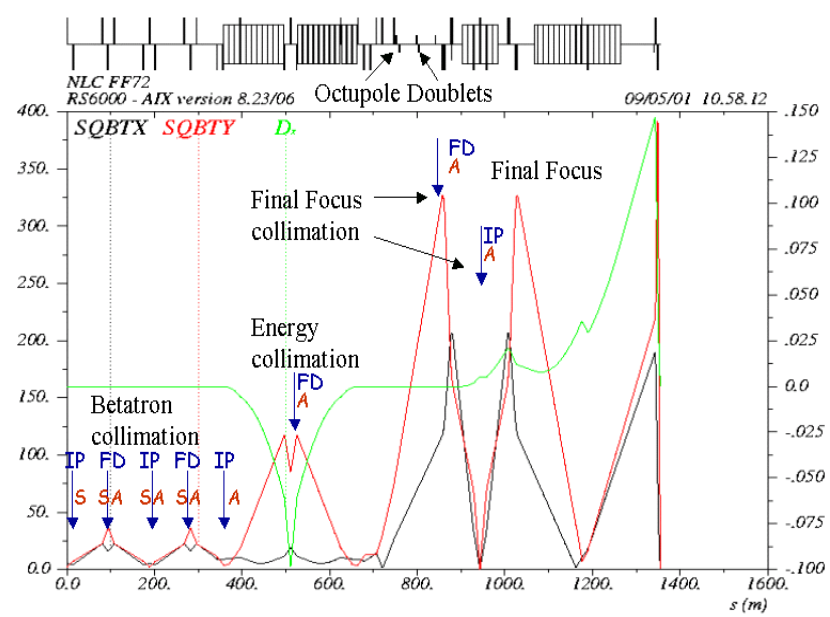

Fig.1: NLC Beam Delivery System Optics

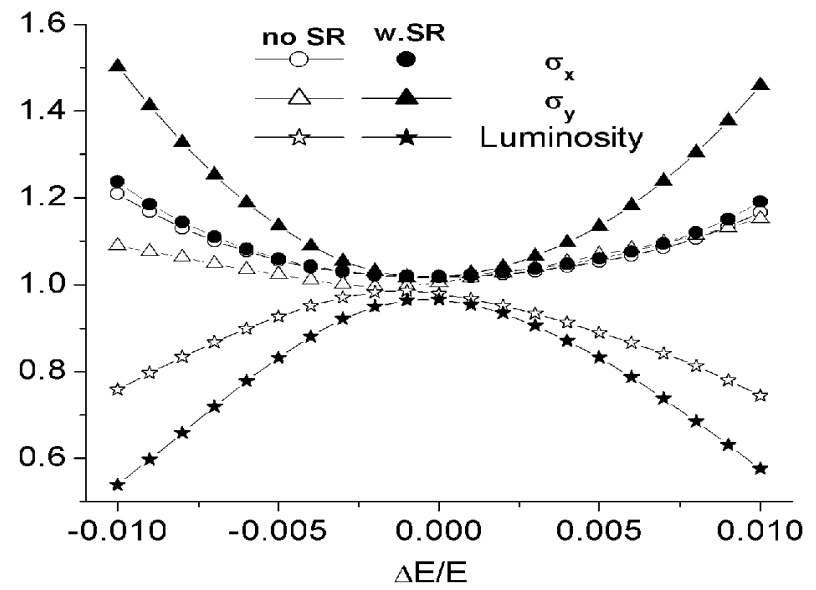

Fig.2: NLC Beam Delivery System luminosity and spot sizes vs the beam energy 

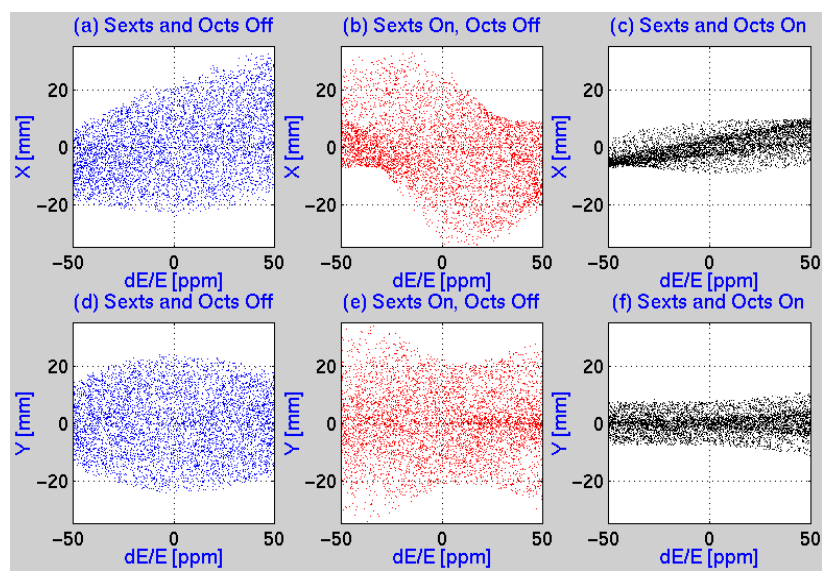

Fig.3: Horizontal and vertical beam distribution in the FD at the peaks of $\beta_{x}$ and $\beta_{y}$ respectively, vs. the beam energy. Input flat beam distribution with maxima $\left(\sigma_{x}, \sigma_{x}\right.$, $\left.\sigma_{y}, \sigma_{y^{\prime}}\right)=(24 \mu \mathrm{m}, 1.0 \mathrm{mrad}, 5.4 \mu \mathrm{m}, 4.4 \mathrm{mrad})$, the beam dimensions are given assuming the IP $\beta$ 's.

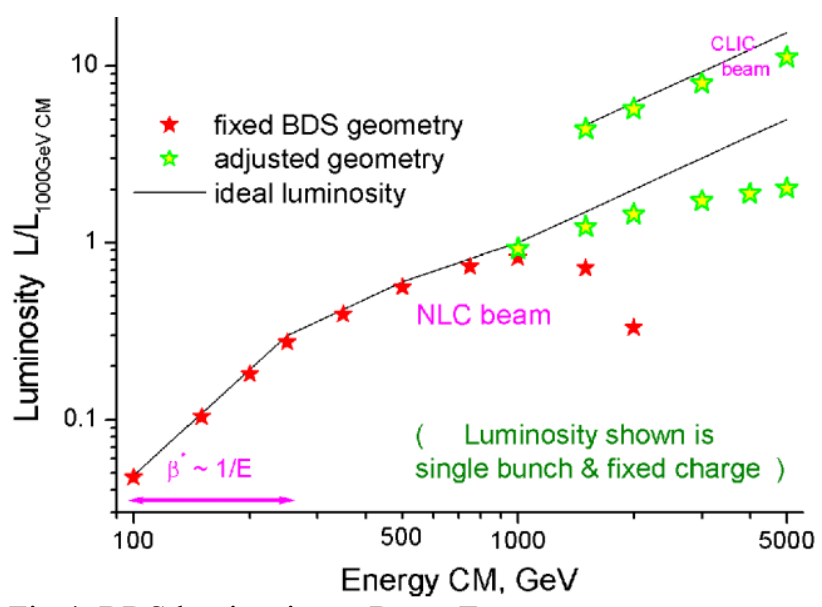

Fig.4: BDS luminosity vs Beam Energy

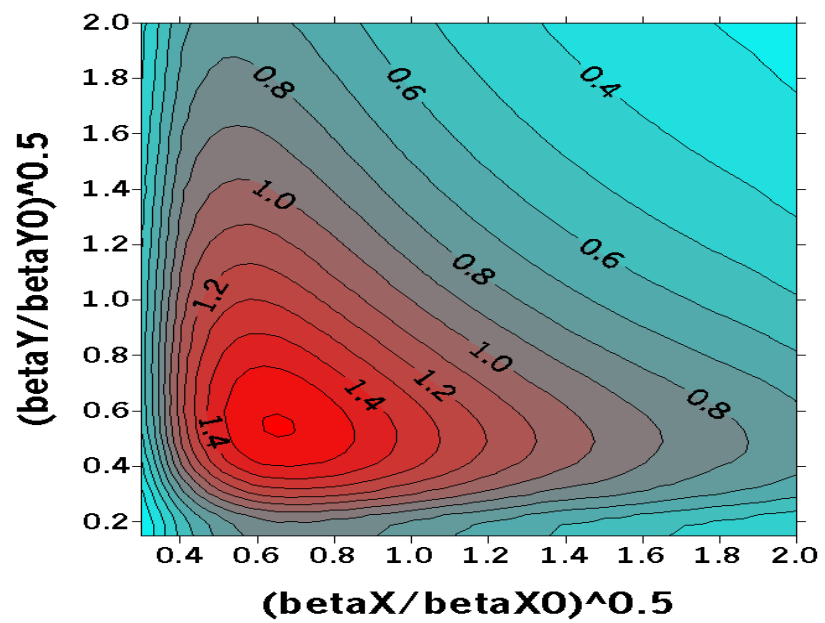

Fig.5: Dependence of the luminosity on the IP $\beta$ functions. The curve labeled 1.0 corresponds to the Tab.1 design luminosity. The hour-glass effect and beam-beam effects are not taken into account.

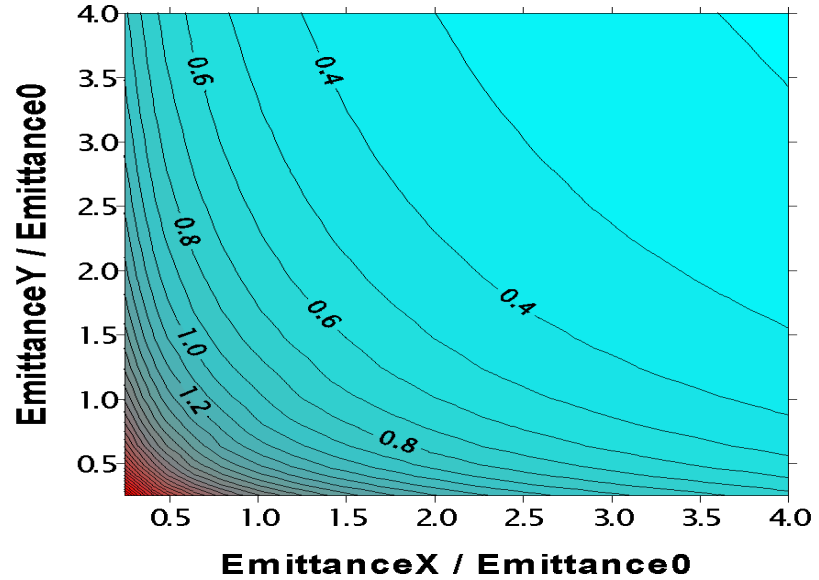

Fig.6: Dependence of the luminosity on the beam emittances. The curve labeled 1.0 corresponds to the Tab.1 design luminosity. The hour-glass effect and beambeam effects are not taken into account.

\section{TOLERANCES AND TUNING}

The tolerances and tuning of the FF have also been extensively investigated. Fig.7 shows the tolerances on quadrupole motion corresponding to $2 \%$ luminosity loss. The FD tolerances are the tightest, because any motion of the FD steers the beams out of collision. The rest of the tolerances are of the order of $100 \mathrm{~nm}$, as long as the IP position is stabilized by a feedback system. Fig. 8 shows the tolerances on magnet field errors for $2 \%$ luminosity loss. The tolerances were computed by FFADA [10].

The procedure for optimizing the luminosity of a nonideal system with errors has also been simulated. Fig.9 shows the luminosity as a function of field strength errors, with and without the use of standard'tuning knobs'to optimize the luminosity. The knobs used are linear combinations of sextupole movers to orthogonally adjust each of the possible first order aberrations: waist position, dispersion and coupling. The sextupoles strengths are also reoptimized. It seems that near optimal performances can still be achieved with field error of the order of $10^{-3}$. Such magnitude of errors can be easily detected with lattice diagnostics techniques like the one used in FFTB [4].

In the tuning simulation presented in this paper, we used an improved program MONCHOU [5] which can perform particle tracking, include ground motion and field errors, and can perform tuning with arbitrary defined knobs to optimize luminosity.

Even for a perfect system, the luminosity can degrade over time due to ground motion moving the magnets. This magnet motion is modeled accordingly to the 'ATL Law' [6], with an assumed magnitude for the drifts similar to the one measured at SLAC [7]. The effect of this motion on luminosity with and without corrections is shown in Fig.10. Feedback to correct the trajectory and to optimize the luminosity with first order aberration knobs, similar to techniques used at SLC $[8,9]$, can maintain the optimal performance of the system for nearly one year, 
even for large cumulative drifts of the magnet positions. Eventually a beam-based quadrupole alignment procedure should be reapplied.

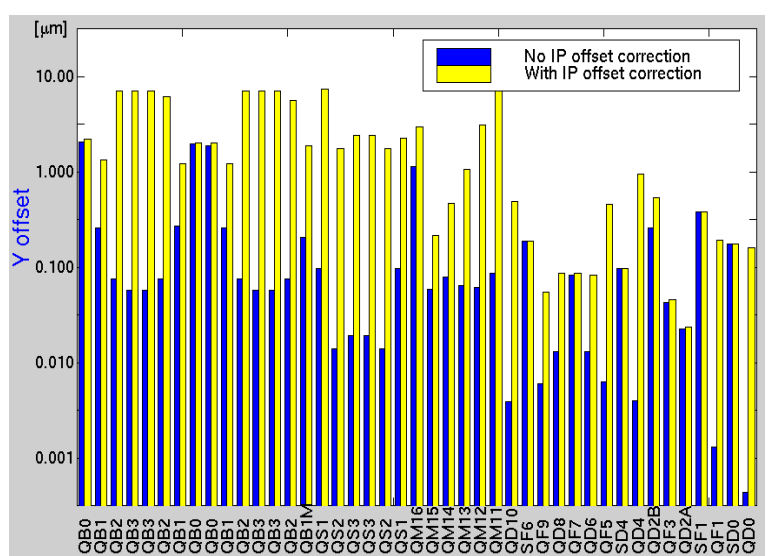

Fig.7: BDS vertical quadrupole position tolerances for a $2 \%$ luminosity loss.

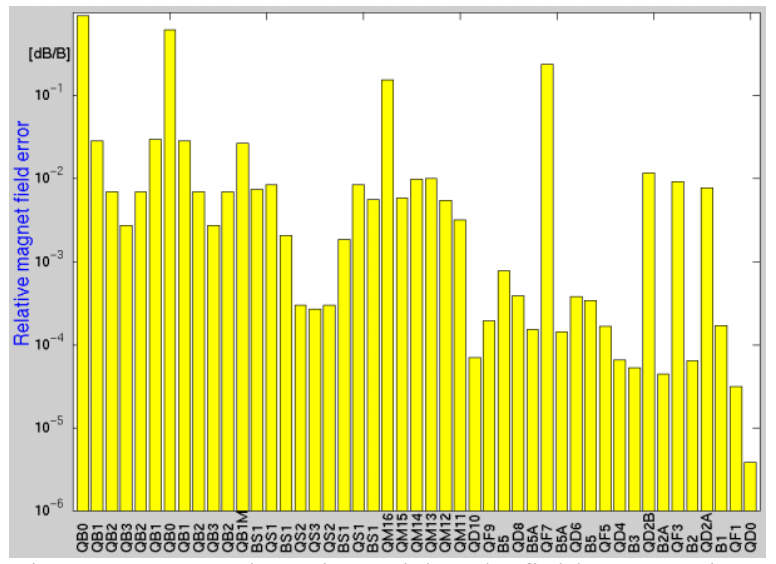

Fig.8: BDS quadrupole and bends field error tolerances for a $2 \%$ luminosity loss.

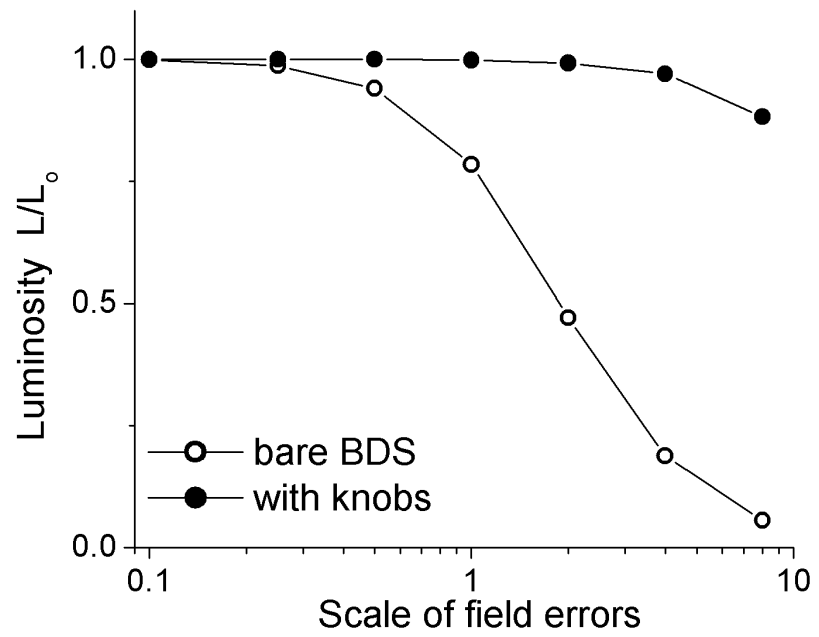

Fig.9: BDS luminosity degradation due to random quad field errors as a function of the error magnitude. The error scale is the ratio with respect to the values in Fig. 8

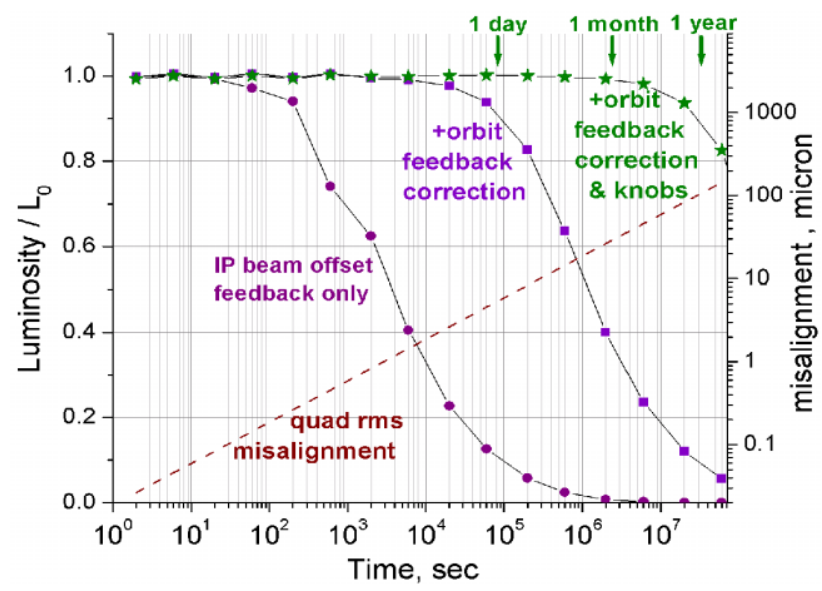

Fig.10: BDS luminosity degradation due to quad motion vs time. The value of the ATL coefficient is assumed to be $A=510^{-7} \mu \mathrm{m}^{2} / \mathrm{m} / \mathrm{s}$.

\section{CONCLUSIONS}

The new Final Focus design meets or exceeds the required performance for the NLC. Simulations indicate that the system has the necessary correction elements so it can be tuned to maximize luminosity even in the presence of errors. The system is relatively insensitive to magnet motion so the luminosity can be maintained for extended periods with only feedback. These properties should allow the luminosity delivered by a real system to be very close to the theoretical optimum.

\section{REFERENCES}

[1] P.Raimondi, A.Seryi, SLAC-PUB-8460, 2000; "New Developments for Final Focus Systems", LINAC2000 Proc; Phys. Rev. Lett 86, 3779 (2001).

[2] P.Raimondi, A.Seryi, "Halo Reduction by means of non linear optical elements in the NLC Final Focus Systems", PAC2001 Proc.

[3] The CLIC study team, "A $3 \mathrm{TeV}$ e+e- Linear Collider Based on CLIC Technology", CERN 200008 .

[4] By V. Bharadwaj, et al., In Proc. of PAC 95, vol. 2, p.752-754, 1995.

[5] A.Seryi, The MONCHOU program, 1996-2001.

[6] B. Baklakov, et al. Tech. Phys. 38, 894 (1993).

[7] A.Seryi, SLAC-PUB-8597, 2000, and refs. therein.

[8] T. Himel, et al., PAC93; SLAC-PUB-6125 (1993).

[9] L. Hendrickson, et al., in Proc. of ICALEPCS 97, Beijing, China; SLAC-PUB-8027, 1999.

[10] O.Napoly, B.Dinham, "FFADA, computer design of final focus system for linear colliders", EPAC94, London, UK; S.Fartoukh, O.Napoly, "Second-order error calculation package for beam transfer lines", EPAC98, Stockholm, Sweden. 